\title{
Closed Loop Synchronous Buck Converter with Variable Input Voltage
}

\author{
Kapil Jangid ${ }^{1}$, Medha R. Giri ${ }^{2}$ \\ ${ }^{1,2}$ Department of Electrical Engineering, Rajiv Gandhi College of Engineering and Research, Nagpur
}

\begin{abstract}
Power electronic converters play an important role in many electronic circuits used in portable application. This paper proposes a PWM based synchronous buck converter with constant input voltage as well as variable input voltage which is designed for low voltage and high efficiency applications. The variable voltage to converter is indicating the implementation with PV energy. This report will give the detailed description of synchronous buck converter, their working modes, simulation circuit along with results.
\end{abstract}

Keywords: synchronous buck converter, constant current feedback control, pulse width modulation, variable input voltage.

\section{Introduction}

DC-DC converters are some of the simplest power electronic circuits which convert one level of electrical voltage into another level by switching action. These converters have received an increasing deal of interest in many areas. This is due to their wide applications like power supplies for personal computers, office equipment, appliance control, telecommunication equipment, DC motor drives, automotive, aircraft, etc. The analysis, design, control and stabilization of switching converters are the main factors that need to be considered. Many control methods are used for control of switch mode DC-DC converters and the simple and low cost controller structure is always in demand for most industrial and high performance application. Each control method has its own advantages and drawbacks, and its effectiveness is determined by the application where it's applied.

This paper deals with the closed loop implementation of synchronous buck converter with constant current at the load voltage. Section 2 of the paper describes the operation of synchronous buck converter, section 3 explains the calculation part of converter, section 4 consists of simulations and results.

\section{Operation of Synchronous Buck Converter}

Figure 1 shows the circuit configuration of the synchronous buck converter which converts unregulated DC to regulated

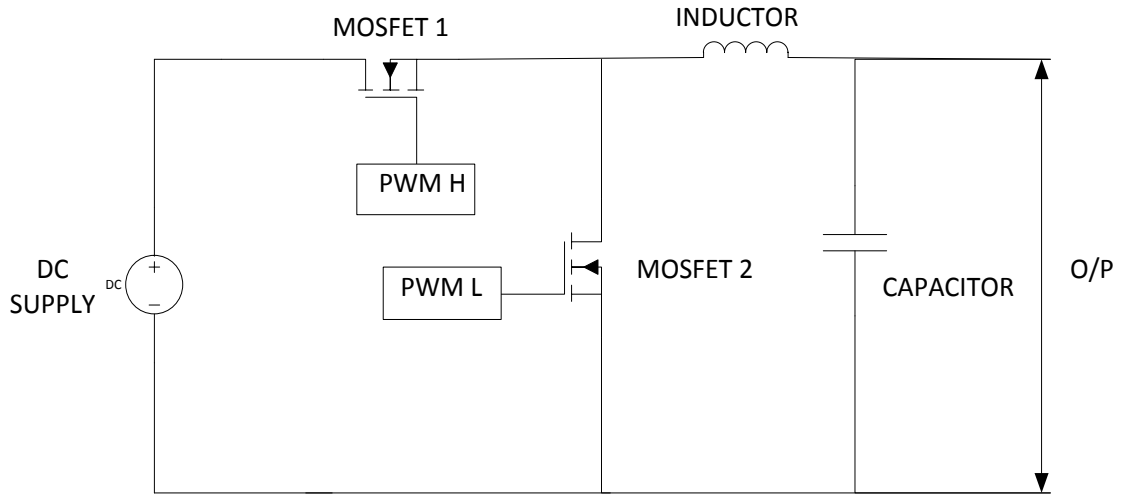

Fig. 1 Synchronous Buck Converter

DC. The chopper is operated by turning the MOSFET $1 \mathrm{ON}$ and OFF at a high switching frequency. Whenthe MOSFET 1 is turned ON, the voltage Vinis also applied across the MOSFET 2, but gate pulses are missing in order to turn ON MOSFET 2. Triggering pulses required for both MOSFETs are complementary to each other shown in figure 2. Hence at any point of time only one MOSFET will get activated. The ON state of MOSFET 1 always implies the OFF state of MOSFET 2. With the MOSFET 1 turned ON, current IL beings to build up. The growth of IL occurs exponentially due to the inductance L. The MOSFET 1 is kept ON for a time interval TON, and OFF for the interval TOFF. At the instant when MOSFET 1 is turned OFF, current has a 
finite value which is the peak value of the output current during the first chopper cycle. This peak current occurs at the instant the MOSFET 1 turns OFF. The presence of the inductance prevents the sudden drop of current to zero. The decay of currentcauses an induced voltageto appear across the inductance. Because of the complementary pulses, the MOSFET 2 conducts and causes the current flow to continue and decay exponentially.

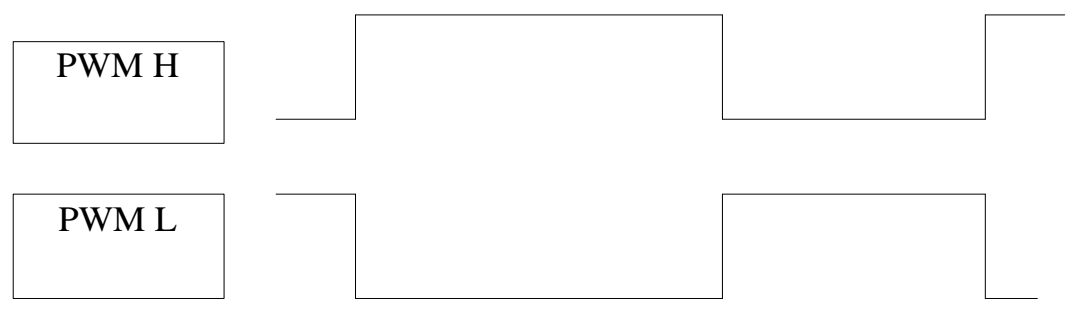

Fig. 2 Complementary Triggering Pulses

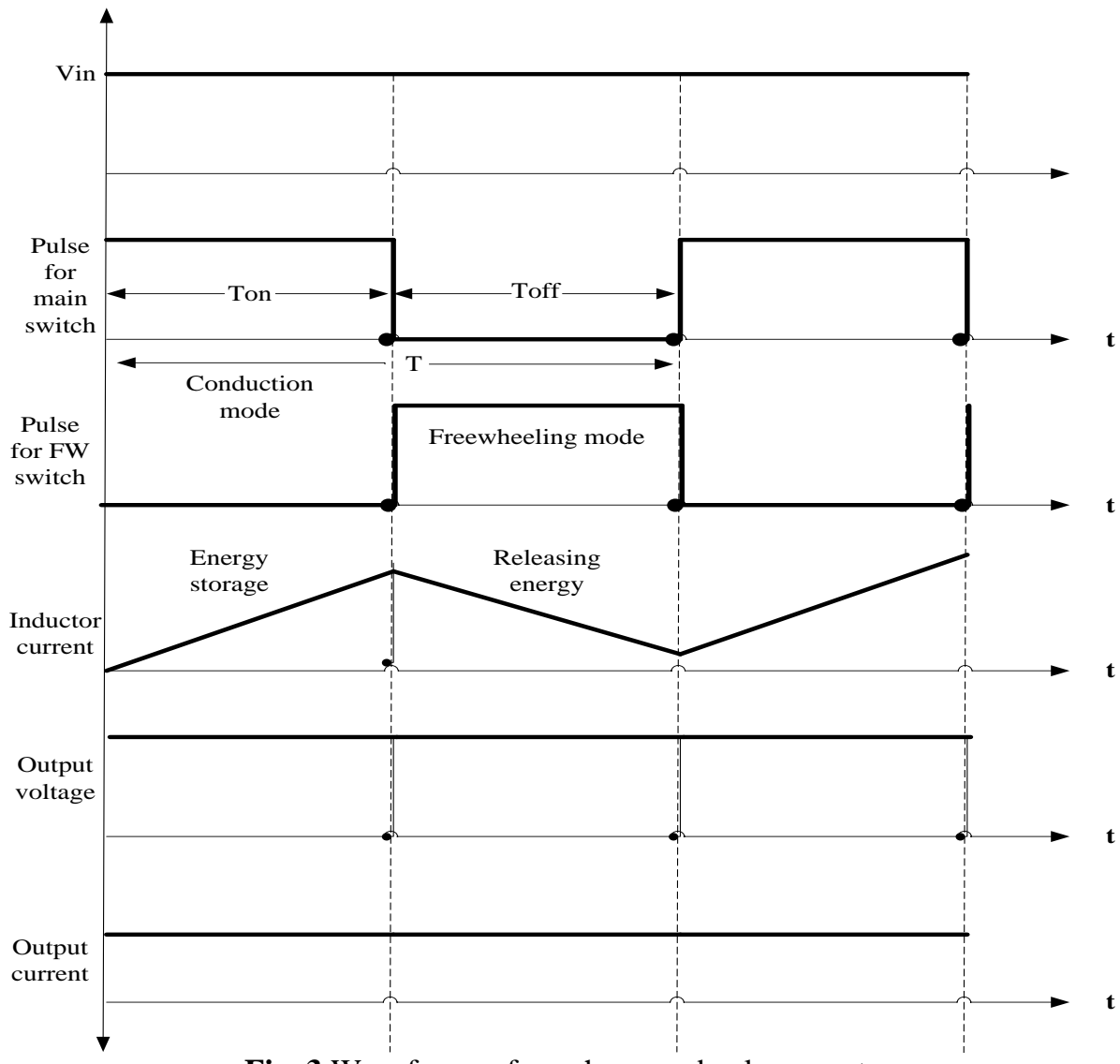

Fig. 3 Waveforms of synchronous buck converter

The term Freewheeling is commonly used to describe the flow of current in this manner without the aid of a voltage source, but solely due to the stored energy in the inductance. Hence MOSFET 2 is called as Freewheeling MOSFET.The purpose of the MOSFET 2is to provide the freewheeling path for the MOSFET 1 when it is turned OFF.The decay of currentcontinues as long as MOSFET 1 remains OFF, that is, for a duration TOFF. The lowest value of decaying current for the first chopper cycle is shown in the figure 3 . The second chopper switching cycle commences when MOSFET 1 is turned ON again at the end of the first TOFF, and the current again starts to build up. Due to the initial current, the second peak will be larger than first peak. Consequently, the process continues and gives the step down voltage. The synchronous buck converter is more efficient as compare to conventional buck converter because the diode in conventional buck converter has more conduction losses as compare to switching losses of MOSFET 2. But the relation between the input voltage Vin, output voltage Vout and duty cycle $D$ remains same as that of conventional buck converter which is given by:

Vout $=$ Vin $\times D$

(1) 


\section{Calculations of Synchronous Buck Converter}

The synchronous buck converter consists of an unknown inductor and capacitor as shown in figure 1 . The calculations of these unknown parameters are performed by assuming some other parameters as shown in table 1.

Table 1. Assumed parameters

\begin{tabular}{|l|l|}
\hline PARAMETERS & VALUES \\
\hline Input Voltage Vi & $40 \mathrm{~V}$ \\
\hline Output Current Vo & $21 \mathrm{~V}$ \\
\hline Load Current Io & $700 \mathrm{~mA}$ \\
\hline Duty Ratio D & 0.525 \\
\hline Switching Frequency Fsw & $100 \mathrm{kHz}$ \\
\hline Output Ripple Current $\Delta \mathrm{Io}$ & $15 \%$ of Io i.e. $115 \mathrm{~mA}$ \\
\hline Output Ripple Voltage $\Delta$ Vo & $50 \mathrm{mV}$ \\
\hline Output Capacitance ESR & $0.06 \Omega$ \\
\hline Input Capacitance ESR & $0.3 \Omega$ \\
\hline ESL & 0 \\
\hline
\end{tabular}

\section{A. Calculation of Inductor}

The voltage across the inductor is given by equation 2 ,

$V=L \frac{\Delta I}{\Delta T}$

By rearranging the above equation,

Therefore from table 1 ,

$$
L=\left[(V i-V o) \cdot\left(\frac{D}{F s W}\right)\right] / \Delta I o
$$

$$
\begin{gathered}
L=\left[(40-21) \cdot\left(\frac{0.525}{100000}\right)\right] / .115 \\
L=0.868 \mathrm{mH}
\end{gathered}
$$

\section{B. Calculation of Capacitor}

The voltage for capacitance is given by equation 3 ,

$\Delta V o=\Delta I o \cdot\left[E S R+\left(\frac{\Delta T}{C}\right)+\left(\frac{E S L}{\Delta T}\right)\right]$

By putting values from table 1 the above equation becomes,

Therefore, by rearranging,

$$
\Delta V o=\Delta I o .\left[E S R+\left(\frac{\Delta T}{C}\right)\right]
$$

$$
\begin{gathered}
C=(\Delta I o . \Delta T) /[\Delta V o-(\Delta I o . E S R)] \\
C=\frac{0.115 \times 75.25 \times 10^{-6}}{[0.05-(0.115 \times 0.06)]} \\
C=14.01 \mu F
\end{gathered}
$$

\section{Simulation and Results}

The arrangement for variable input voltage is shown in the figure 4 which mainly consists of a constant block, repeating sequence block, summer and controlled DC voltage source.

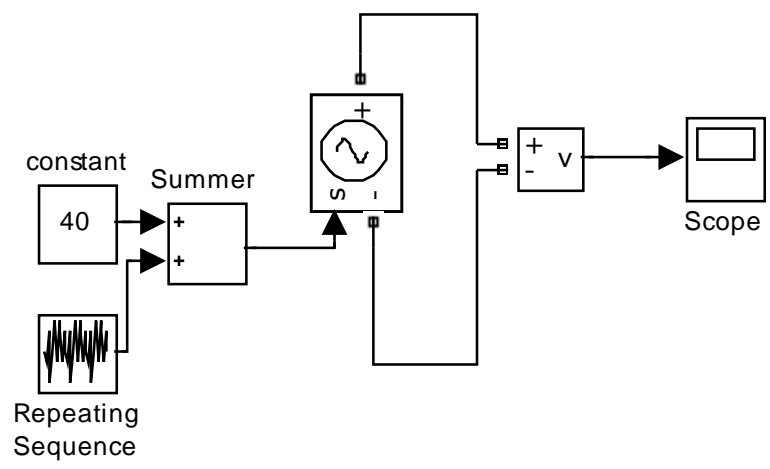

Fig. 4 Variable input voltage arrangement 
The result for variable input voltage is shown in fig. 5

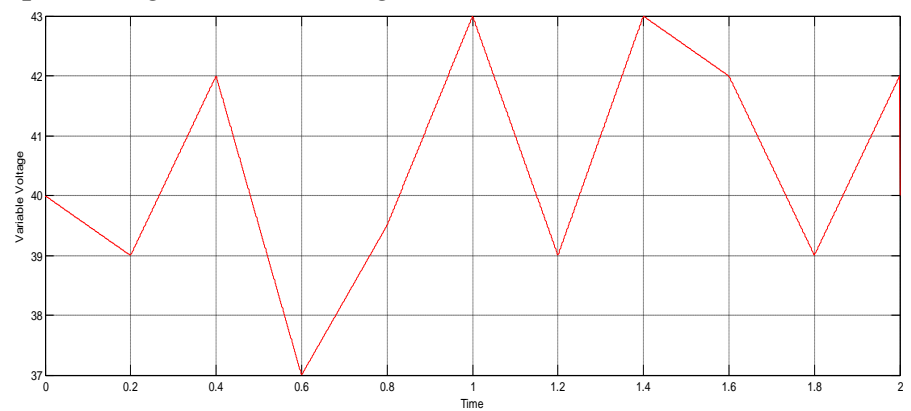

Fig. 5 Variable input voltage

The Closed loop simulation of synchronous buck converter is shown in figure 6 which is maintained constant at $700 \mathrm{~mA}$ of load current. The values of $\mathrm{Kp}$ and $\mathrm{Ki}$ are 0.9 and 0.02 in the PID controller. The calculated values of inductor and capacitor are considered and all the other parameters are shown in table 1 . The R L load is considered as $30 \Omega$ and $1 \mathrm{mH}$.

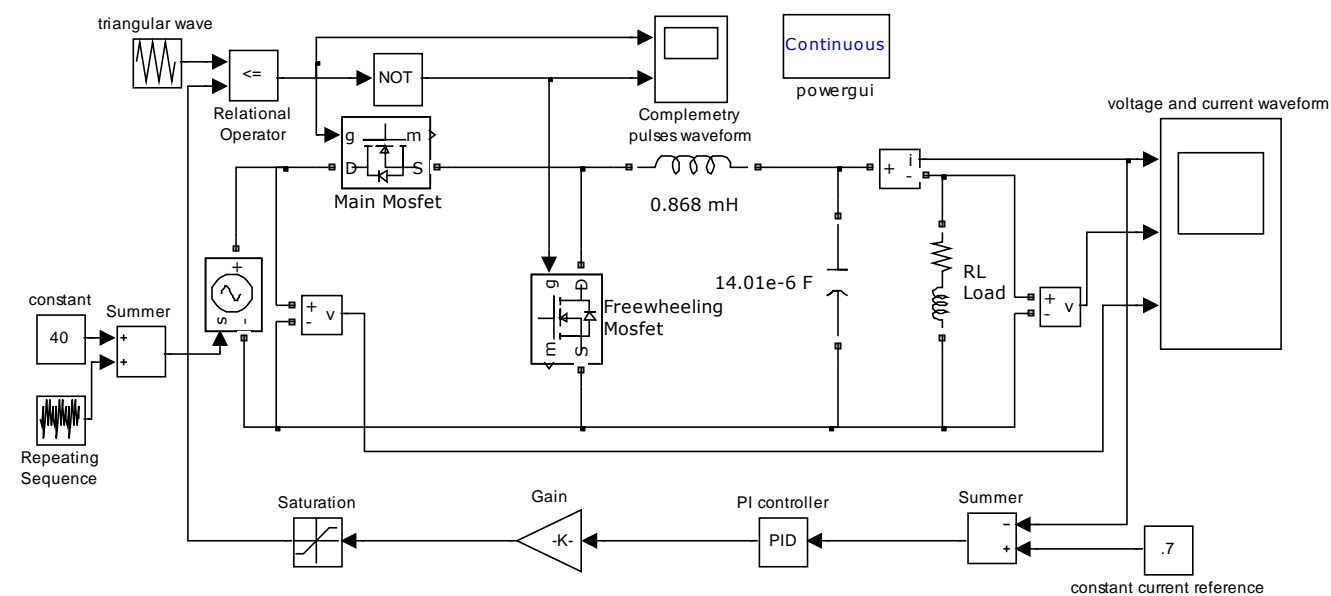

Fig. 6 Closed Loop Synchronous Buck Converter

The current feedback is taken from current measurement which is compared with the constant current reference by using summer block. The error is generated and fed to PI controller with the value mentioned above. The corrected error is then attenuated in order to maintain the peak to peak value of triangular wave generator. The comparison of error signal and triangular wave is done by using relational operator block and hence pulses at $100 \mathrm{kHz}$ are obtained.
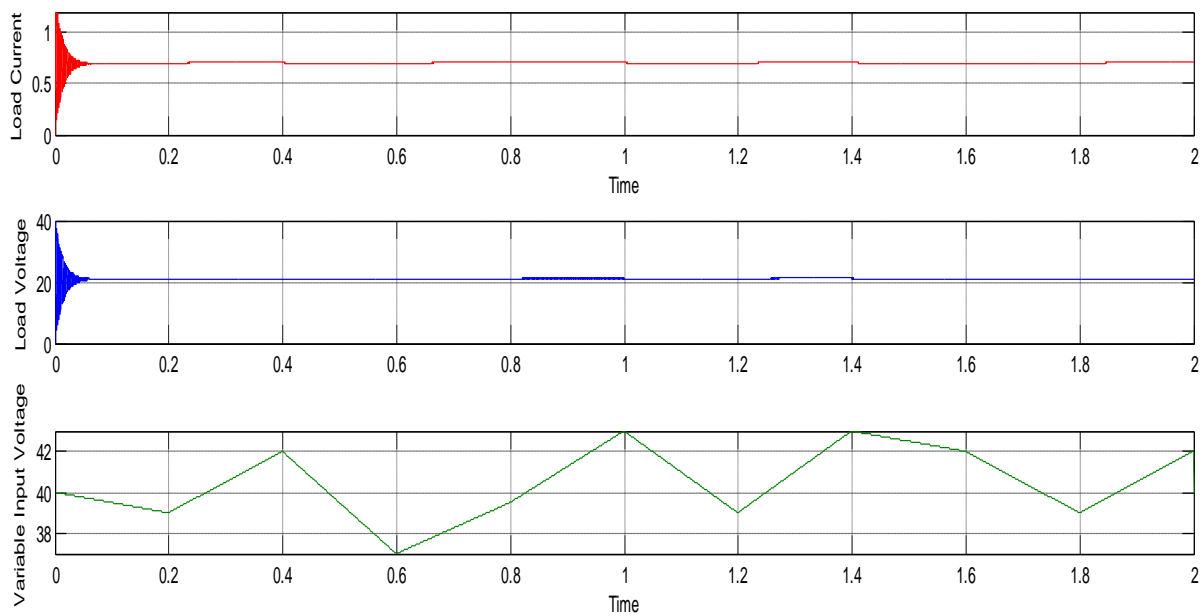

Fig. 7 Results of synchronous buck converter 
From the figure 7 it is clearly seen that the voltage and current are maintained at constant value with the change in the input voltage. Hence synchronous buck converter is working properly in a closed loop maintaining load current at $700 \mathrm{~mA}$.

\section{Conclusion}

In this paper, a control technique is proposed with the synchronous buck converter for maintaining constant current at the load side. As the switching frequency goes on increasing the rating magnetic components like inductor and capacitor goes on decreasing which makes the circuit lighter which is an absolute advantage but with this increment in switching frequency problem of noise arises. So maintaining an optimal frequency could be the option.

\section{References}

[1] S. -Y. Fan, S. -Y. Tseng, Y. -J. Wu and J. -D. Lee, "PV Power System Using Buck/Forward Hybrid Converters for LED Lighting,"IEEE Energy Conversion Congress and Exposition, pp. 2584-2591, Sept. 2009.

[2] Huang-Jen Chiu, Yu-Kang Lo, Jun-Ting Chen, Shih-Jen Cheng, Chung Yi Lin, and Shann-ChyiMou, "A High Efficiency Dimmable LED Driver for Low-Power Lighting Applications,"'IEEE Transactions on Industrial Electronics, vol. 57, no. 2, pp. 735743, February 2010.

[3] Hongjia Wu, Yongliang Zhang, Menglian Zhao, HongfengShen and Xiaobo Wu, "A Constant-on-Time Based Buck Controller with Active PFC for Universal Input LED,’Power Electronics and Drive Systems (PEDS),pp.551-556, June 2015.

[4] A.S.Werulkar and P.S.Kulkarni, "Design of a Constant Current Solar Charge Controller with Microcontroller based SoftSwitching Buck Converter for Solar Home Lighting System,” 2012 IEEE International Conference on Power Electronics, Drives and Energy System, pp. 1-6, Dec. 2012.

[5] RohanDayal and Leila Parsa, "Non-isolated Topologies for High Step-down Offline LED Driver Applications," IEEEApplied Power Electronics Conference and Exposition (APEC), pp. 988-993, Feb. 2012. 